Pak. J. Agri. Sci., Vol. 56(3), 775-784; 2019

ISSN (Print) 0552-9034, ISSN (Online) 2076-0906

DOI: $10.21162 / P A K J A S / 19.8036$

http://www.pakjas.com.pk

\title{
WEED DETECTION IN WHEAT CROP USING UAV for PRECISION AGRICULTURE
}

\author{
Ahmed Mateen ${ }^{1,2, *}$ and Qingsheng Zhu ${ }^{1}$ \\ ${ }^{1}$ College of Computer Science, Chongqing University, China; ${ }^{2}$ Computer Science Department, University of \\ Agriculture Faisalabad, Pakistan \\ *Corresponding author's e-mail: ahmedmatin@hotmail.com
}

\begin{abstract}
Agriculture plays a significant role in overall GDP of any country. So, there are several efforts those are being made to develop and increase the crop yield. In any of crop, weed i.e. (unwanted crops) is the major concern that may leads to poor production of crop. Therefore, an automatic crop monitoring system is required to monitor the weed. This system will help the farmers to monitor the crops, in gradual fashion, once it has been cultivated. Then specific Vegetation Index (VI) would be applied to locate all green portions in the image that would be part of early wheat crop or weed patches. We used Object Based Image Analysis (OBIA) algorithm to detect the weed patches in RGB and Multispectral imagery captured by UAV at 30-60 m altitude is used to acquire the images of wheat fields. Once the weed patches successfully identified from between the crop rows and within the crop rows then connected component-based classification technique is used that successfully classify the detected object either the object is related to weed patches or actual crop. The core objective of this work is to lessen the human involvement and to introduce the latest techniques and computation technology, peculiarly to identify the weed patches within the crop rows as well as between the crop rows in wheat field. Moreover, exploitation of UAV technology is also the core objective that will significantly provide the site specifically herbicides spraying.
\end{abstract}

Keywords: Precision Agriculture, Object Based Image Analysis, Vegetation Index, unmanned aerial vehicle (UAV), polygonbased thresholding

\section{INTRODUCTION}

Digital image processing is extensively used in several areas of research to develop the self-governing systems. Recently computer vision and digital image processing attracts the attentions of researchers from different areas of research. Hence the field of agriculture is one of them ((Xiang et al.,2011). Currently, precision agriculture (PA) is providing various solutions in different agriculture domains. Moreover, this remote sensing is also an active area of research which is also using digital image processing approach to solve complex problems (Lottes et al., 2017; Comba et al., 2015). Although many of advancements have been done in this area but still there are numerous issues those are addressable pertaining to crop management, early pest detection, weed patches detection (Sankaran et al., 2016). Therefore, development of automatic weed detection system is considered as an active concerning issue to be resolved accurately (Wu et al., 2011). Weed is unwanted crops, grown automatically with wanted crop (Pérez-Ortiz et al., 2015). Weed patches are undesirable plants those are self-grown and not under the farmer control (Tang at al., 2016). This uncontrolled weed patches may lead to poor crop yields and dense utilization of pesticides (Tanget et al., 2016). Furthermore, Unmanned Arial Vehicle (UAV) will be used to capture the field images, by RGB camera mounted on it, of early wheat crop with varying illuminations (Guerrero et al., 2013). In addition to this, distinct altitude of UAV flight is also considered for better results. The major advantage of exploiting the UAV technology over the satellite imagery, as it can fly at desired level of altitudes that may provide a flexibility in capturing the ultra-high resolutions images of different crop fields. This may also lead to attain more accurate solution to detect the different weed species in varying crops (Torres-Sánchez et al., 2015). By extract the green by converting the RGB three band image to one band color image (Tiede et al., 2010). once the image weed is detected with by using polygon-based thresholding technique further it is also system requirement to check if the detected objects belong to wanted crops or unwanted crops (Ji.,2011). Hence, computer vision strategy, connected component is applied to detect the overall pattern objects (Yang., 2015). As it is also supposed, in many cases weed patches mostly exhibit random pattern and at early stage weed is significantly different in color and texture as compared to actual crop. Furthermore, the input image is converted into binary image which is the output of previous step (de Castro., 2018). Then, the text extraction process is in progress of two sub images. At first each text elements are bounded one by one separately. All connected components are then bounded by a green line (Tang., 2017). unmanned aerial vehicle (UAV) and unmanned ground vehicle (UGV) plays significant role in this 
regard. As both technologies are vastly used in many areas with varying applications with regard to perspective of problem domain. There are several issues, pertaining to PA and PF those can be solved using UAV and UGV. The gradual monitoring and inspection crop is the major area of concern which may be solved using above mentioned technologies (Sarkar et al., 2015). Furthermore, this may lead to monitor and control of weed infections, crop illness, crop row alignment and weed patches detection etc (Vega et al., 2015). Thence by introducing the weed robotics and artificial intelligence in the area of agriculture for different problems provide massive benefits toward easiness in farming and crop yield enhancement (Buayaui et al., 2017). Although, there are still many challenges those must be solved to provide fully automated solution for precision agriculture industry (Chen $e t$ al., 2018).

(Comba et al., 2015) observed new altitude record was set in 1998 by the slightly larger Pathfinder-Plus (PF+). the significantly larger solar-powered UAV Helios became the highest-flying propeller-driven aircraft in the world, soaring to $96,000 \mathrm{ft}$ (Ganesan et al., 2014). These high- altitude milestone flights were all conducted in the restricted airspace of the Pacific Missile Range Facility (PMRF) at Barking Sands, Kauai, Hawaii. Development and integration of a solar energy storage system is currently in progress (Gnädinger et al., 2017). The future vision is that Helios and similar aircraft will function as 11-mile-high towers capable of sustained long-term flight, while supporting wireless communication and imaging systems (Herrera et al., 2014). All types of vegetation indices can be accurately applied on UAV based imagery and suitable results can be obtained once the vegetation fraction is successfully separated from images (Vikhe et al., 2016). This segregation of vegetation fraction from background and other non-vegetation objects appeared in images may useful for detection of weed patches in herbaceous crops at early stage (Sankaran et al., 2015). This allows cultivation of more number of plants. The problem arises from the fact that both intended plants and weeds demonstrate the same green color which makes differentiation a tedious task. In that case, the credible way to resolve this issue consist of manual identification of the plants in agricultural fields by a seasoned and experienced farmer. However, this manual detection process of weeds is time consuming and the accuracy of the identification process may be subjected to human errors (Tang et al., 2017). In most of the instances (Pérez-Ortiz et al., 2015) field presence of weeds is usually determined keeping in view many determinant factors with respect to extracted features such as, color, shape, length, aspect and length ratios. In order to differentiate weeds from plants, different researchers have utilized diverse types of classifications algorithms. Among all, the Support Vector Machine (SVM) has been regarded as the most commonly used machine learning algorithm for identification of weed or infected regions existing in an agricultural filed (Gnädinger et al., 2017). In one study by subdivision of the input images was carried with in to different cells followed by the subsequent application of SVM for identification of only those regions which comprised of crop plants. Despite the significant developments with regard to weed identification, the aforementioned techniques possess very low suitability for detection of weed regions overlapped with crop plants said that the differentiation of wanted crops and unwanted weed patches at the early stage of development requires images with different spatial resolution and illumination (Torres-Sánchez et al., 2015). Although, different UAV and camera technologies produces different results, but for weed detection task it is highly required to attain high resolution images with high pixel density. On the basis of some reports related to precision agriculture many of researcher's claims that satellite or UAV based data must be taken at specific moment in order to attain the high degree of accuracy in detection process. Moreover, it is also recommended to use UAV technology for image acquisition, satellite instead as it provides data at distinct altitudes by considering environmental implications in this regard. Despite to this, there are several other factors those provide robust evidence to use adopt UAV for precision agriculture issues. It requires some simple steps for one-time flight of UAV for data acquisition process (Buayaui et al., 2017). In addition to benefits of UAV discussed above, all types of vegetation indices can be accurately applied on UAV based imagery and suitable results can be obtained once the vegetation fraction is successfully separated from images. This segregation of vegetation fraction from background and other non-vegetation objects appeared in images may useful for detection of weed patches in herbaceous crops at early stage (Gandhi et al., 2015). Vegetation indices provides an authentic mechanism to separate vegetation objects specifically while working on precision agriculture and remote sensing domains. There are several Vis those can be applied differently on distinct nature of images. All types of VIs are based upon some pixel based arithmetic operations to extract spectral information from the radiation reflected by green objects, crops in this case. In this context class of image is very much important before applying any of VI as many of indices perform mathematical operations on RGB images more accurately. On the other hand, high resolution images are considered good for weed detection task, but classification requires some significant features of an image. With regard to image resolution and pixel depth, there are various types of images captured thorough UAV's. Very high scale resolution images represent challenges for several operation of OBIA (Torres-Sánchez et al., 2015). There are more pixels required to represent the object in images more appropriately, which makes the algorithms computationally intensive. On the other hand, these images exhibit more intera-class variations in terms of spectral information (Vega et al., 2015). While it is required to deal with variation in spectral space, OBIA is 
considered as one of the suitable choices. Furthermore, OBIA technique is quite flexible as it works on objects in image those are based upon similar homogenous group of pixels, which constitute objects. Thereof, it reduces all the problems due to intra-class spectral variability which is emerged due to nature of object. The core idea behind this algorithm is to group-up the pixels those spatially adjacent to each other and then constituting the object by combining the homogenous pixels and applying classification techniques (Comba et al., 2015).

\section{MATERIALS AND METHODS}

This research provides a robust and computational inexpensive procedure to detect the weed patches between and within the crop rows specifically in wheat crops. As it is also described in the previous chapter that the core challenge in identifying the weed patches in actual crop fields is the spectral similarity between crop and weed patches. As up to certain period when the wheat seed is inseminated there is no existence of weed but after one or two months it appears with the same color and texture of wheat. Hence, this research solves this problem in the context of object-based image analysis (OBIA) by integrating the machine learning algorithms with color-based threshold technique to extract the weed features and identifying the pattern of weed. This system will alleviate the excessive intervention of user to manually estimate the crop health in gradual fashion. Although, there are various approaches used to develop the OBIA about the context of problem. As, OBIA is exploited to separate the combination of pixels with homogeneous properties and connected pixels with similar spectral information, which lessen the spectral variation due to crop textures, shadows and other similar aspects. The core idea behind this process is to combine the similar pixels pertain to some object, and further applying the classification algorithm using the extracted features in previous process. Before applying the proposed OBIA stages, the quality resolution of acquired image data is very much important and require significant attention to further achieve results with high degree of accuracy. Thereof, attaining the images with high spectral information of whole field, orthomosaicing is an essential step to initiate the detection process. Orthomapping is considered one of the crucial steps in remote sensing. As it requires standard images with distinct elevation, altitudes and spectral information. Following are the main steps to those are adopted to for detection process.

There are many of previous series of DJI Phantom, as Phantom 3 and Phantom 2, and all these Phantom series quadcopter drones provide various flight timings in different modes. From a certain test, it is authenticated that the Phantom 4 gives maximum flight timing and this flight timing is very much useful comparatively other quadcopter. Specification for tools and software are describe in Table 1-2.
Table 1.Specifications of Phanto 4 DJI Commercial Drone

\begin{tabular}{lll}
\hline Sr\# & Specifications & \\
\hline 1. & Weight of Quadcopter & 1380 grams \\
& Inclusive of Batteries & \\
2. & Maximum Flight Speed & $20 \mathrm{~m} / \mathrm{s}$ in Sport Mode \\
3. & Flight Timing & $20 \mathrm{minutes}$ approx. \\
4. & Obstacle Sensing System & Yes (Rang 0.7 to $25 \mathrm{~m}$ ) \\
5. & Vision Positioning System & Yes (Effective range $0-10 \mathrm{~m}$ ) \\
6. & Slow Motion Video & Yes (Resolution $1920 * 1080$ \\
& Recording & @ $120 \mathrm{fps}$ ) \\
7. & Battery Capacity in & $5350 \mathrm{mAh}$ \\
& Intelligent Flight Mode & \\
8. & Maximum Accent and & $6 \mathrm{~m} / \mathrm{s}$ \\
& Descent Speed & $4 \mathrm{~m} / \mathrm{s}$ \\
\hline
\end{tabular}

Once the orthomosaic image is generated next step is to crop the certain portion of image to further segregate the wanted and unwanted crops. This operation is randomly performed using MATLAB R2016b. And greenness is quantified from cropped image. There are several mechanisms those are being used to successfully extract the green portion in certain crop image. In this research, excess green index is used to fully extract the vegetation from given image. There are several other mathematical mechanism those may help in this regards. This vastly used method for identification of vegetation fraction is solely based upon visible spectral-index, excess green index, by negating excess red index from excess green, the vegetative index. Finally, excess green is extracted which is significantly vegetation. This process of extraction may encounter some problems pertaining to shades of green color which may include light green to dark green. Following equations \# 1 \& 2 peculiarly describe the Excess Green (ExG) index.

$$
\operatorname{ExG}(2)=2 g-r-b
$$

Where the $\mathrm{r}, \mathrm{g}$ and $\mathrm{b}$ are red, blue and green channels respectively.

$$
E x G R=E x G-E x R=E x G-1.4 r-g
$$

\section{- Excess Green}

$$
\operatorname{ExG}(2)=2 g-r-b
$$

Where $r, g$ and $b$ represent the red, green and blue channels of RGB color space.

These VIs were developed to emphasis the green component of vegetation image. Moreover, these indices were also designed to handle the problems of detection due to variability of variation in daylight illumination. Thereof, the understanding of the applications is that every mathematical expression can be converted back to its original form(Ameer et al.,2018). Hence, the VIs are able to convert the images from the original RGB three-band space to a greyscale one. Hence by using applying the excess green index on the cropped image is shown in figure 1 . As it is quite clear from the output that every color is segmented with different shades. The input image was comprised of varying shades green channels, as weed was slightly different in color as compared to actual crops. 
Table 2. List of Tools \& Software.

\begin{tabular}{|c|c|c|c|}
\hline Sr. & Tools/Software & Version & Component/Module \\
\hline$\overline{1 .}$ & DroneMapper Rapid & v1.1 20180323 & Orthomapping for GIS \\
\hline 2. & eCognition & eCognition Developer 9.0 & In Built Rule Set \\
\hline 3. & MATLAB R2017b & $\mathrm{R} 2017 \mathrm{~b}$ & $\begin{array}{l}\text { Image Processing ToolboxComputer Vision Toolbox } \\
\text { Image Acquisition Toolbox }\end{array}$ \\
\hline 4. & ImageJ & $1.52 \mathrm{a}$ & Histogram, Color Spreads \\
\hline 5. & UAV & DJI Phantom 4 & \\
\hline 6. & RGB Sensor & TCS34725 & \\
\hline 7. & Tetracam NIR Sensor & 230218 & \\
\hline
\end{tabular}

- Color Index of Vegetation

CIVE $=0.441 r-0.881 g-0.385 b+18.78745$ (4)

Where $r, g$ and $b$ represent the red, green and blue channels of RGB color space. As the color index is simply extracted using CIVE index.

\section{- Excess Green Minus Excess Red}

$$
E x G R=E x G-E x R=E x G-1.4 r-g(5)
$$

As the Equation 5 represents the difference of excess green index $(\mathrm{ExG})$ and excess red (ExR).

Further, which clearly separates the green channels with varying pixel values of green. As it is described every type VI yields different results depending upon the image type and nature of capturing device. Hence, all these mathematical equations are applied in MATLAB environment and following results are generated. For output validation, UAV flight is adjusted at varying altitudes. All these vegetation fraction quantifications are exploited on various altitudes just to calculate the optimum accuracy. As in this research the UAV flight altitude is fixed $20-30 \mathrm{~m}$ altitude is used to acquire the images of wheat fields. The drone was not programmed to fly and capture images automatically, but the images are taken manually using remote control. Thereof the images are taken where the maximum vegetation is seen though drone camera. The high resolution of the acquired image data provides an ease in the visualization of individual vegetation component. Hence, the output of every vegetation index is subject to the quality of image in terms of resolution and color scheme. Once the vegetation indices are successfully applied on the field imagery the further step is to successfully extract the weed patches by applying the suitable threshold techniques. The images, undergoes some vegetation fraction quantification technique, can be used for thresholding either for automatic of manual one. In this research, all the algorithmic steps are being employed on RGB color space but with minor variations these can also be applied on multispectral imagers those also comprises of near infrared band as well. There are several ways of applying thresholds techniques. Same as the indices, threshold also provides different results with regard to varying images. As the suitable threshold is applied, next step is to combine the resultant image for classification purpose. Although, the multispectral imagery is subject to near infrared band, but there are various studies those have suggested to not use the NIR images instead. As the results obtained by RGB images are more accurate and valid for further processing. Instead of using the multispectral imagery all the algorithmic steps are exploited on RGB images and yields the optimal results along with enhanced classification performance as well. However, the study has also pointed out some crucial aspects about the selection of band for better segmentation and weed quantification. There are several tools and software with varying versions specifications those can be used for distinct but similar purpose. Thereof, in this research, following list of tools (see table 2) are being used to develop to manipulate different stages of OBIA algorithm.

\section{Algorithm for weed detection: Below is our proposed} model algorithm for automatic weed detection

1. Load image data set

2. Perform of orthomap with Rapid DrownMapper

3. Binary Masking of orthomap with matlab bw $=$ createMask $(\mathrm{ROI})$

4. Terrain generation 3D image in matrix level

5. Crop Map portion is performed $\mathrm{J}=$ imcrop (I, rect)

Rect is vector [x, y, width, height]

$\mathrm{X}$ represent color pixel representation

$\mathrm{Y}$ represent frequency of occurrence of certain pixel value

6. Histogram of cropped image

Imhist(I)

Define Region of Interest (ROI)

7. Color conversion of ortho image - operation performed with eCognition Developer 9

Color verification of high green and its variations for classification of weed.

8. Vegetation Indices

Excess green

$\operatorname{ExG}(2)=2 g-r-b$

Color Index of Vegetation

$C I V E=0.441 r-0.881 g-0.385 b++18.78745$

Excess Green minus Excess Red

$E x G R=E x G-E x R=E x G-1.4 r-g$

$\mathrm{BW}=\left\{\begin{array}{l}1 \text { if } \mathrm{VSI}>\mathrm{T} \\ 0 \text { otherwise }\end{array}\right.$ 
where VI $€\{$ ExG; CIVE; ExGR $\}$ and $T$ is the threshold value.

9. Polygon thresholding and RGB masking

FOR $i$ from 1 to (row_length-1):

IF gray-value $\left(\right.$ pixel $\left._{i}\right)-$

gray-value $\left(\right.$ pixel $\left._{i+1}\right)>$ edge - Threshold THEN

Set pixel $_{i}=$ WHITE

ELSE

Set pixel $i=B L A C K$

END IF

END

- Data set is loaded and orthomap is created for scale uniformality of UAV photos (by merging orthophotos scaling and cartography information)

- Binary masking is applied to the above orthomap which will convert the image in black and white (foreground and background).

- Next step is to perform crop map portion for 3dimensional view of terrain map, resulting image has characteristics of other dimension which is height for vegetation and non-vegetation pixels. It is easy to crop portion of terrain map to 3D map.

- Color histogram is made to analyze color spread of RGB across different pixels.

- Next step is color conversion segmentation is applied as thresholding become easy on color base segmentation.

- Then applied vegetation indexing and polygon thresholding, after polygon selection with certain portion of image by automatic selection of color. Binary mask identifies objects with pixel values less than the selected threshold.

- Binary masking is applied to the above orthomap which will convert the image in black and white (foreground and background).

- Next step is to perform crop map portion for 3dimensional view of terrain map, resulting image has characteristics of other dimension which is height for vegetation and non-vegetation pixels. It is easy to crop portion of terrain map to 3D map.

- Color histogram is made to analyze color spread of RGB across different pixels.

- Next step is color conversion segmentation is applied as thresholding become easy on color base segmentation.

- Then applied vegetation indexing and polygon thresholding, after polygon selection with certain portion of image by automatic selection of color. Binary mask identifies objects with pixel values less than the selected threshold. wheat field using UAV at varying altitude (Ameer et al., 2018).

Table 3 provides the frequency band ranges of RGB and NIR images.
Table 3. Band Ranges for RGB \& NIR Images

\begin{tabular}{lll}
\hline Sr. & Band Type & Band Range \\
\hline 1. & Blue & $440-510 \mathrm{~nm}$ \\
2. & Green & $520-590 \mathrm{~nm}$ \\
3. & Red & $630-685 \mathrm{~nm}$ \\
4. & Red Edge & $690-730 \mathrm{~nm}$ \\
5. & Near Infra-Re & $760-850 \mathrm{~nm}$ \\
\hline
\end{tabular}

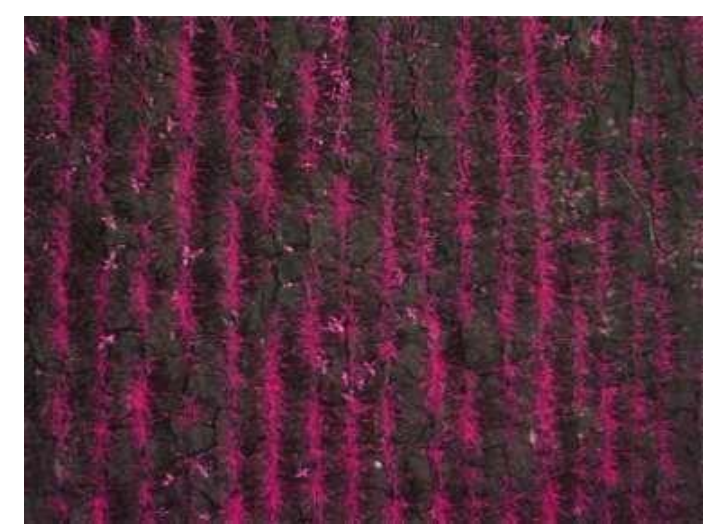

Figure 1. Visible weed patches in multispectral image.

Once the input images are given to generate orthomap, initially Rapid Dronemapper converts all the images into and binary image which is actually used to estimate the 3D elevation of generated map (Ameer et al.,2018). It processor intensive operation, as it consumes the most of computation resources once the process is initiated. At the start of process, all the images are combined, and simple binary mask is generated that is further used for orthomap creation. This process is completed in three iterations and each iteration yield some specific output. As it is clearly shown in binary mask of all the images is generated that will further decide the elevation of crop height at varying positions. This orthomosaicing process provides the map output as 3D bird eye view. Therefore, generated map output can be depicted at desired angle of elevation. In this experiment the generated binary mask is at 120 degrees, which will further decide to create the map at same angle. Moreover, this binary mask generation also plays a significant role while terrain development that gives a 3D like view of overall field. Once the binary mask is successfully created by the Rapid Drone mapper, next is to create terrain in grayscale. As it creates point cloud at each pixel by considering the actual images from which this map is created, and the binary mask created previously.

Creating combined and complex single image from satellite or aerial images, captured by UAV is a fundamental step in orthomosaic generation. With the ease in availability of high quality imagery; the extensive application of UAVs in different domains; and by introducing latest trends and techniques of digital photography, orthomosaics are created from large number of images specifically captured at low- 
altitude. Thereof, the more images are required to compose the composite map to specify the land covered by vegetation. Finally remarkable attention has been paid to develop methods to automatically generation of orthomosaics from aerial or satellite images acquired by UAV and satellite respectively. Following Figure 2 shows the terrain automatically generated by Rapid Dronemapper.

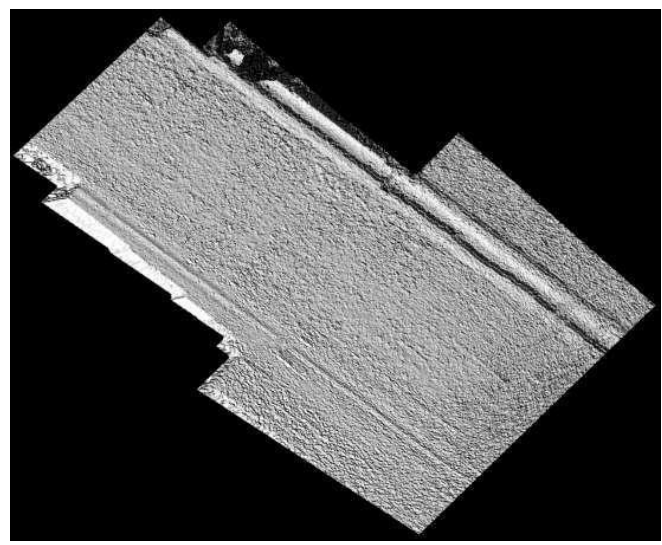

Figure 2. Terrain Image from Masked.

A typical processing pipeline for automated orthomosaic generation can be summarised as follows:

Digital images are captured, generally along with navigation data - usually the GPS location of the camera at the time each image was captured, but possibly also camera orientation from inertial sensors. Structure-from-motion techniques are applied to generate a terrain model, along with the camera locations and orientations. Alternatively, an existing terrain model can be used (Ameer et al.,2018). The images are projected onto the terrain model, creating an orthoimage for each input photograph. These images are mosaiced in such a way as to minimize the visual transition from one image to the next. The proposed formula compensated the uniformity in RGB color space to some extent, while the color difference variation was not smooth. Hence the shown RGB color space can be further exploited for threshold -based segmentation of vegetation objects. Once the RGB color space is used, the system provides the flexibility in implementation of threshold in manual fashion.

Binary Masking of Vegetation portion: Once the polygon is selected the next step is find the binary mask of certain objects shown in image. This mask can be further used in application of segmentation of these objects with the pixel's values less than the selected threshold (Ameer et al.,2018). Binary mask is also used while generating the actual output of RGB image once it undergoes the threshold. Thereof, this step provides the output as logical array, which can be shown as binary image in Figure 3.
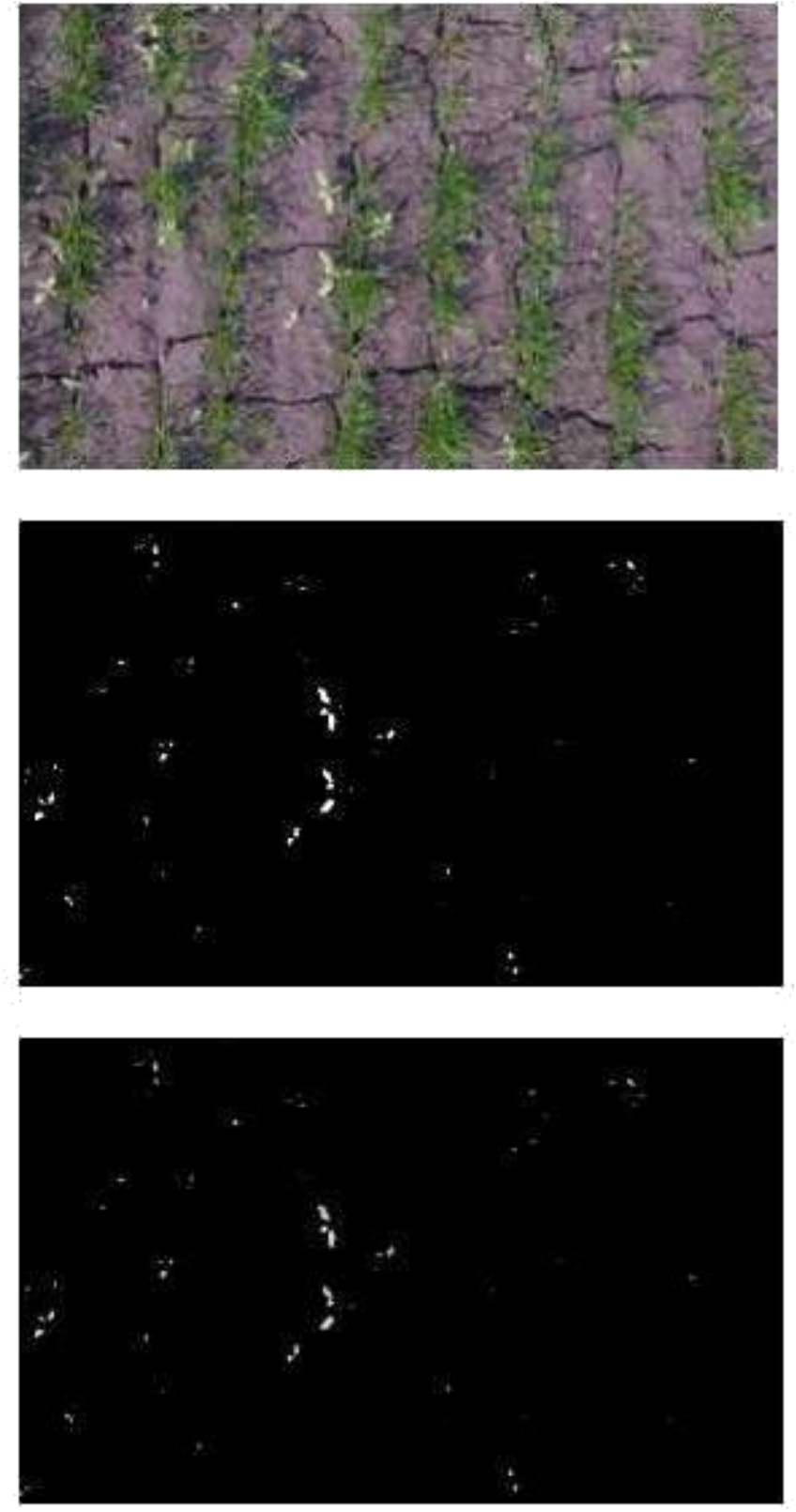

Figure 3. Combined output of binary mask and segmented image

orthomosaic image is generated with the help terrain and 2 D binary mask generated by software (Ameer et al.,2018). Therefore, generated mosaicked image is not only the combination of three -color channels but also provides another dimension which the height of vegetation and nonvegetation pixels at each point is. These phenomena yields three dimensional like depiction of orthomosaic field map. Hence, it is quite easy to crop the certain portion of terrain map comparatively three-dimensional map. As this task is 
accomplished using MATLAB random manual crop functions with the help rectangle.

The overall color variations of color in weed image can be analyzed using color histograms of that image. Image histogram represents the overall spread color across different pixels. Because in case of the true color or RGB image, one pixel is represented by three variations of Red, Green and Blue channel. Figure 4 shows the overall result histogram of cropped image. The value of color pixel is represented on $\mathrm{x}$ axis, while the frequency of occurrence of that certain pixel value is depicted on y-axis. Moreover, it's also quite clear that the wheat image massively comprises of green pixels, therefore the range of pixels 75 to 125 is depicted on peaks. Hence, this is the major area of interest for further analysis.

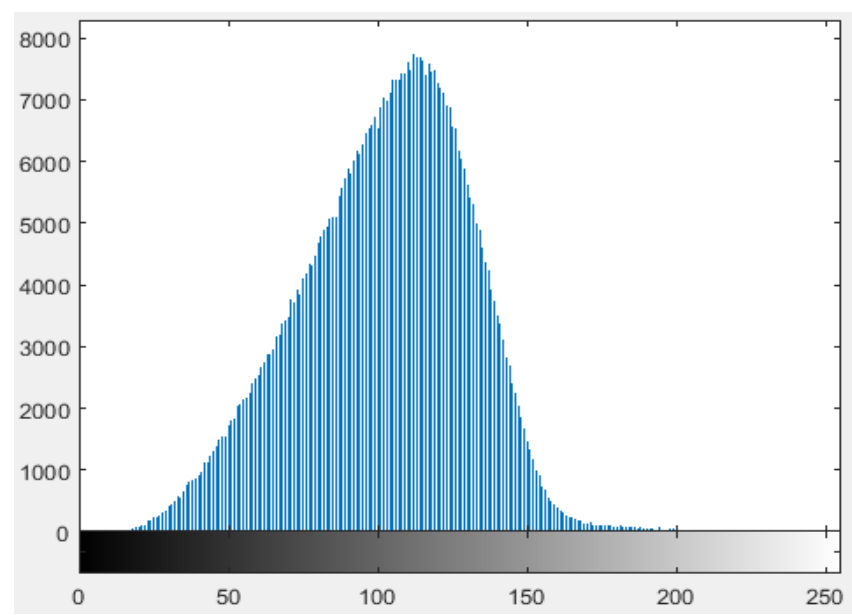

Figure 4. Color Spread of RGB Image

\section{RESULTS}

In this experiment, wheat crop is major concern to detect the weed at early stage. Following images are taken from of smaller pixel size as seen in Table 4. So, there was decrease in proportion of pixels which were representing bare soil and vegetation . Regarding agronomic point of view, this result is called positive because proper weed control should be done earlier to achieve effective results in post-emergence period value obtained were 0.40 and 0.38 . At $30 \mathrm{~m}$ flight, there was a better vegetation index automatic thresh holding, became . were obtained at lower altitude . At flight altitude of $60 \mathrm{~m}, \mathrm{R} 2$.

Table 5. Binarization accuracy of proposed methodology compared to other approach

\begin{tabular}{lcccc}
\hline Image \# & \multicolumn{3}{c}{ Vegetation Segmentation Accuracy \% } \\
\hline & $\begin{array}{c}\text { Proposed } \\
\text { Methodology }\end{array}$ & Otsu & Watershed & ANN \\
\hline (1) & 88.45 & 77.23 & 25.37 & 61.78 \\
$(2)$ & 70.84 & 60.44 & 42.18 & 70.67 \\
$(3)$ & 91.55 & 79.91 & 66.78 & 88.23 \\
$(4)$ & 98.42 & 45.12 & 20.28 & 26.38 \\
$(5)$ & 96.02 & 26.78 & 32.67 & 40.23 \\
$(6)$ & 73.55 & 65.24 & 58.96 & 44.84 \\
$(7)$ & 83.13 & 28.67 & 21.54 & 29.34 \\
$(8)$ & 97.91 & 61.35 & 56.78 & 41.12 \\
\hline
\end{tabular}

The different methodologies of color transformations are naturally limited by the aspect of conversion from color transformation to content-based image styling. One major color distribution scheme can also be employed in some cases, but this type of color distribution often cannot provide perfect results for all types of image contents (Ameer et al., 2018).

In Figure 5 it depicted (Torres -Sánchez et al.,2015) that at flight altitude of $30 \mathrm{~m}$, excellent result was obtained regarding automatic vegetation detection. R2 value of 0.72 and 0.63 Comparison with other approaches and performance analysis : The proposed technique segmentation performance is evaluated by comparing the accuracy gained for the binarization process between the suggested methodology and other well-known methodologies. These methods are generally regarded

Table 4. Provides the brief overview of specification of image

\begin{tabular}{|c|c|c|c|c|c|}
\hline Sr\# & Image Type & Number of channels & Resolution & Average Altitude & Wavelength $(450-515 \mathrm{~nm})$ \\
\hline 1. & True Color & $\begin{array}{l}\text { (Bit Depth }=24 \\
\text { bits per pixel) } \\
\text { Red (R) } 8 \text { bits } \\
\text { Green }(G) 8 \text { bits } \\
\text { Blue (B) } 8 \text { bits }\end{array}$ & $5000 \times 4000$ & 30 meters above the ground & \\
\hline 2. & Multispectral & Bit Depth $=24$ bits & $2048 \times 1536$ & 30 meters above the ground & $\begin{array}{l}750-900 \mathrm{~nm} \text {, is used primarily for } \\
\text { imaging vegetation. }\end{array}$ \\
\hline
\end{tabular}

Table 6. Accuracy in crop row detection and its standard error. Results are showed for the UAV flights at every date and flight altitude.

\begin{tabular}{lcccccc}
\hline Altitude (m) & \multicolumn{5}{c}{ DAS } \\
\cline { 2 - 6 } & $\mathbf{3 5}$ & $\mathbf{4 3}$ & $\mathbf{4 9}$ & $\mathbf{6 0}$ & $\mathbf{6 8}$ & $\mathbf{7 5}$ \\
\hline 30 & $83.72 \pm 1.48$ & $83.80 \pm 1.73$ & $85.01 \pm 1.33$ & $76.84 \pm 4.52$ & $74.14 \pm 4.21$ & $80.18 \pm 1.84$ \\
60 & $82.90 \pm 1.58$ & $80.86 \pm 1.74$ & $83.54 \pm 1.60$ & $64.65 \pm 7.59$ & $79.94 \pm 2.72$ & $81.44 \pm 1.99$ \\
\hline
\end{tabular}


color -index -based procedures that generate special grayscale images from the RGB images input. Then, using threshold detection, binary vegetation images are shown in Figure 6. Comparing VF for $30 \mathrm{~m}$ and $60 \mathrm{~m}$ altitude UAV flights with produced. The colour index methods employed include ExG, ExGR, and CIVE selected based on their accurate performance Table 5 . This performance stability is evaluated by comparing mean and standard deviations of the proposed approach with other methods as shown in Figure 5. Based on the results achieved, the proposed methodology was able to generate binary images of vegetation that distinguish from non-vegetation objects for diverse instances. The mean accuracy for the segmentation method for all tested photos was $87.48 \%$ with a standard deviation of $10.75 \%$. While other well recognized techniques such as Otsu, Watershed and ANN showed varied standard deviation ranges from 18.35 to 21.42, as shown in Figure 7.

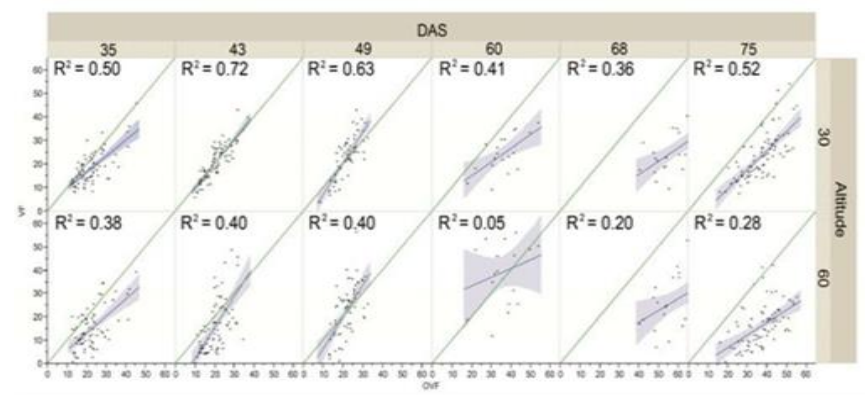

Figure 5. Comparing VF for UAV flights with $30 \mathrm{~m}$ and $60 \mathrm{~m}$.

Segmentation process stability was examined using standard deviation of accuracies for the varied RGB images. It is also recommended to apply the provided methods to segment the gathered images for flight height $40 \mathrm{~m}$ or less, as the higher height images were severely affected by the camera's resolution. Such recommendation is preferable if the desired PA is crop row detection or crop health monitoring. To adopt such a segmentation technique, numerous weed patch identification and weed management applications can be easily deployed. Such suggestion is based on the ability of the proposed segmentation algorithm to handle high-altitude RGB photos, which enable the capacity to cover filed agriculture faster and with less photographs compared to current UAV weed management systems that recommend pictures at $40 \mathrm{~m}$ methodology and other approaches. Std is represented by Table 6 displays the accuracy of crop row detection regarding two flight altitude. At $30 \mathrm{~m}$ altitude, the most accurate date regarding crop row detection were 35,43 \& 49 DAS, while the best dates obtained were $35,45 \& 75$ DAS, when the flight altitude were $60 \mathrm{~m}$.Regarding both flight altitude, accuracy was below $80 \%$ at only 60 and 68 DAS (Torres-Sánchez et al., 2014).

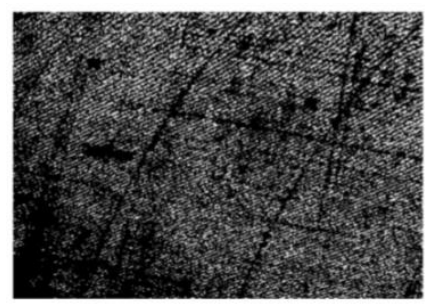

(a)

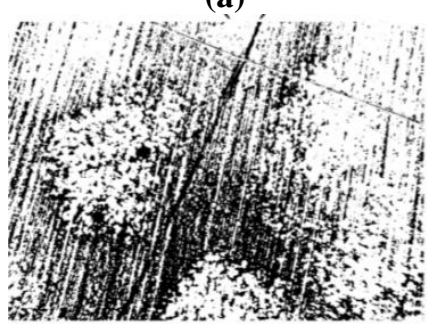

(c)

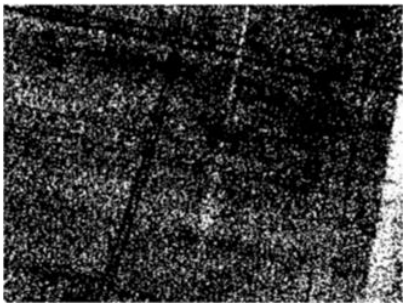

(b)

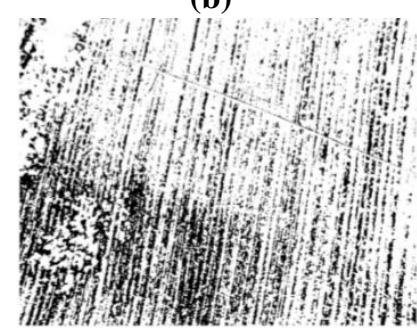

(d)
Figure 6. Generated vegetation binary images a) proposed methodology b) Ostu c) watershed d) ANN

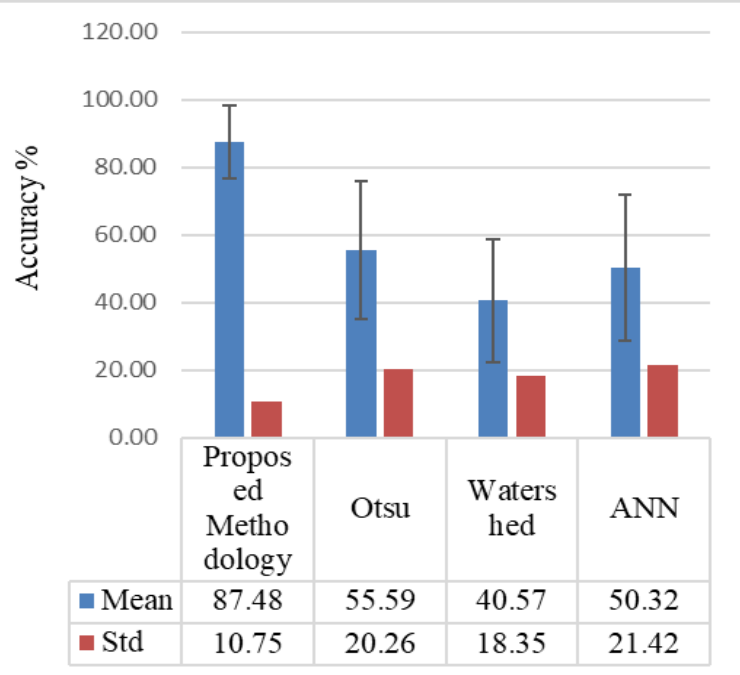

Figure 7. Performance comparison between the proposed

Thus data depicts that the algorithm was implemented successfully for crop row detection in a broad time window. It is important that acquisition time of UAV imagery is increased in days of different weather problems especially in can of wheat because wheat is a winter crop usually and a lot of rainy and windy days may be encountered error bar.

Conclusion: This vegetation index is based upon some mathematical operation performed on each pixel in image. Therefore, each single band of three-color channels is extracted and further mathematical manipulation, pertaining to $\mathrm{ExG}$ is performed to efficiently extract vegetation part. More precisely, any of vegetation index is not only choice to segment vegetation portion from image but image must 
undergo some threshold technique to exclude background lye in certain pixel range. One of the significant advantages of using these vegetation indices is to successfully extract green color channel and then apply some specific thresholding technique i.e. (Otsu threshold etc.) to attain desired results if we are using binary images weed then would be little easy as some of binary segmentation algorithm will do the job effectively Thereof, to attain the maximum accuracy from this algorithm is suggested that crop images must be captured exactly in the early season of the crop, so that directly color based segmentation may be applied to. segment weed patches and actual crop by exploiting any of color index. Moreover another concern pertaining to sliding window-based pixel analysis is that crop rows must be well-aligned while ploughing that will consequently give an effective crop row segmentation while weed detection is performed. Both of above discussed methods, are used to manage large fields with simple UAV equipment. This technology will not only give automatic farm management but also gradual monitoring of farm fields in effective manner.

Acknowledgement: We acknowledge the valuable support of Dr. Muhammad Ahsan Latif and his students in completing this work

\section{RERENCES}

Xiang, H. and L. Tian. 2011. Development of a low-cost agricultural remote sensing system based on an autonomous unmanned aerial vehicle (UAV). Biosyst Eng. 108:174-190.

Lottes, P., R. Khanna, J. Pfeifer, R. Siegwart and C. Stachniss. 2017. UAV-based crop and weed classification for smart farming. IEEE the International Conference on Robotics and Automation (ICRA), pp:3024-3031.

Comba, L., P. Gay, J. Primicerio, and D.R. Aimonino. 2015. Vineyard detection from unmanned aerial systems images. Comput Electron Agr. 114:78-87.

S. Sankaran., L.R. Khot, C.Z Espinoza, S. Jarolmasjed, V.R. Sathuvalli, G.J Vandemark, P.N Miklas, A.H. Carter,

M.O. Pumphrey and N.R. Knowles. 2015. Low-altitude, highresolution aerial imaging systems for row and field crop phenotyping. Eur J Agron 70:112-123.

Wu, X., W. Xu, Y. Song and M. Cai. 2011. A detection method of weed in wheat field on machine vision. Procedia Eng. 15:1998-2003.

Pérez-Ortiz, M., J. Pena, P.A. Gutiérrez, J. Torres-Sánchez, C. Hervás-Martínez and F. López-Granados. 2015. A semi-supervised system for weed mapping in sunflower crops using unmanned aerial vehicles and a crop row detection method. Appl. Soft Comput 37:533-544.

Tang, J., Wang, D., Zhang, Z., He, L., Xin, J. and Xu, Y. 2017. Weed identification based on K-means feature learning combined with convolutional neural network. Comput Electron Agr.135: 63-70.

Tang, J.-L., X.-Q. Chen, R.-H Miao and D. Wang. 2016. Weed detection using image processing under different illumination for site-specific areas spraying. Comput Electron Agr. 122:103-111.

Guerrero,J.M., M. Guijarro, M. Montalvo, J. Romeo, L. Emmi, A. Ribeiro and G. Pajares. 2013. Automatic expert system based on images for accuracy crop row detection in maize fields. Expert Syst Appl 40: 656-664. Ameer, A., M.A. Latif, R. Naseem and M.Z. Iqbal. 2018. Automatic early weed detection in wheat crops using unmanned aerial vehicle (UAV) based imagery. MS (CS) Thesis, Department of Computer Science, University of Agriculture, Faisalabad, Pakistan.

Barrero O., D. Rojas, C. Gonzalez and S. Perdomo. 2016. Weed detection in rice fields using aerial images and neural networks. Paper presented at the Signal Processing, Images and Artificial Vision (STSIVA) XXI Symposium:1-4. 10.1109/STSIVA.2016.7743317.

Gnädinger F. and U. Schmidhalter. 2017. Digital Counts of Maize Plants by Unmanned Aerial Vehicles (UAVs). Remote Sens. 9:544-.

Lameski, P., E. Zdravevski, V. Trajkovik, and A. Kulakov. 2017. Weed Detection Dataset with RGB Images Taken Under Variable Light Conditions. Paper presented at the International Conference on ICT Innovations;PP. 112119.

Meyer, G.E. and J.C Neto, 2008. Verification of color vegetation indices for automated crop imaging applications. Comput Electron Agr. 63:282-293.

Torres-Sánchez J., F. López-Granados and J.M. Peña. 2015. An automatic object-based method for optimal thresholding in UAV images: Application for vegetation detection in herbaceous crops. Comput Electron Agr. 114:43-52.

Tiede, D., S. Lang, F. Albrecht, and D. Hölbling. 2010. Object-based class modeling for cadastre-constrained delineation of geo-objects. Photogramm Eng Rem S. 76:193-202.

Ji, R. and L. Qi. 2011. Crop-row detection algorithm based on Random Hough Transformation. Math Comput Model. 54:1016-1020.

Torres-Sánchez, J., J. Peña, A. De Castro and F. LópezGranados. 2014. multi-temporal mapping of the vegetation fraction in early-season wheat fields using images from UAV. Comput Electron 103:104-113.

Yang, W., S. Wang, X. Zhao, J. Zhang and J. Feng. 2015. Greenness identification based on HSV decision tree. Inform Process Lett. 2:149-160.

de Castro, A, I., J. Torres-Sánchez, J.M. Peña, F.M. JiménezBrenes, O. Csillik and F. López-Granados. 2018. An automatic random forest-obia algorithm for early weed 
mapping between and within crop rows using UAV imagery. Remote Sens. 10:285-.

Tang, J., D. Wang, Z. Zhang, L. He, J. Xin and Y. Xu. 2017. Weed identification based on K-means feature learning combined with convolutional neural network. Comput Electron. 135: 63-70.

Sarkar, S., S. Das and S. S. Chaudhuri. 2015. A multilevel color image thresholding scheme based on minimum cross entropy and differential evolution. Pattern Recogn Lett. 54:27-35.

Vega, F.A., F.C. Ramírez, M.P. Saiz and F.O. Rosúa. 2015. Multi-temporal imaging using an unmanned aerial vehicle for monitoring a sunflower crop. Biosyst Eng. 132:19-27.

Buayaui, P., T. Kantanukul, C. Leung and K. Saikaew. 2017. Boundary Detection of Pigs in Pens based on Adaptive Thresholding using an Integral Image and Adaptive Partitioning. CMU J. Nat. Sci 16:145-156.

Chen, R., T. Chu, J.A. Landivar, C. Yang and M.M. Maeda. 2018. Monitoring cotton (Gossypium hirsutum L.) germination using ultrahigh-resolution UAS images. Precis Agric. 19:161-177.

Ga, G.M., S. Parthiban, N. Thummalu and A. Christy. 2015. NDVI: vegetation change detection using remote sensing and GIS-A case study of Vellore District. Procedia Comput Sci. 57:1199-1210.

Ganesan, P..and V. Rajini. 2014. Assessment of satellite image segmentation in RGB and HSV color space using image quality measures. Paper presented at the Advances in Electrical Engineering (ICAEE); PP.1-5.

Herrera, P.J., J. Dorado and A. Ribeiro. 2014. A novel approach for weed type classification based on shape descriptors and a fuzzy decision-making method. J. Sens. 14:15304-15324.

Vikhe, P. and V. Thool. 2016. Mass detection in mammographic images using wavelet processing and adaptive threshold technique. J Med Syst. 40: 82-.
Pérez-Ortiz, M., J. Pena, P.A. Gutiérrez, J. Torres-Sánchez,

C. Hervás-Martínez and F. López-Granados. 2015. A semisupervised system for weed mapping in sunflower crops using unmanned aerial vehicles and a crop row detection method. Appl Soft Comput. 37:533-544.

Tang, J., D. Wang, Z. Zhang, L. He, J. Xin, and Y. Xu. 2017. Weed identification based on K-means feature learning combined with convolutional neural network. Comput Electron Agr. 135: 63-70.

Torres-Sánchez, J., F. López-Granados and J.M. Peña. 2015. An automatic object-based method for optimal thresholding in UAV images: Application for vegetation detection in herbaceous crops. Comput Electron Agr. 114:43-52.

Pérez-Ortiz, M., P. A. Gutiérrez, J.M. Peña, J. TorresSánchez, C. Hervás-Martínez, and F. López-Granados. 2015. An experimental comparison for the identification of weeds in sunflower crops via unmanned aerial vehicles and object-based analysis. Paper presented In International Work-Conference on Artificial Neural Networks, pp. 252-262.

Vega, F. A, F. C. Ramírez, M. P. Saiz, and F. O. Rosúa. 2015. Multi-Temporal Imaging Using an Unmanned Aerial Vehicle for Monitoring a Sunflower Crop. Biosyst. Eng. 132, 19-27.

Gandhi, N, L. J. Armstrong, O. Petkar, and A.K. Tripathy.2016. Rice crop yield prediction in India using support vector machines. Paper presented In 2016 13th International Joint Conference on Computer Science and Software Engineering (JCSSE) pp. 1-5.

Torres-Sánchez, J. F. López-Granados and J.M. Peña. 2015. An Automatic Object-Based Method for Optimal Thresholding in Uav Images: Application for Vegetation Detection in Herbaceous Crops. Comput Electron Agric. 114: 43-52. 